\title{
On pattern matching with $k$ mismatches and few don't cares
}

\author{
Marius Nicolae ${ }^{\mathrm{a}, *}$, Sanguthevar Rajasekaran ${ }^{\mathrm{a}}$ \\ ${ }^{a}$ Department of Computer Science and Engineering, University of Connecticut, 371 \\ Fairfield Way Unit 4155, Storrs, CT 06269, USA
}

\begin{abstract}
We consider the problem of pattern matching with $k$ mismatches, where there can be don't care or wild card characters in the pattern. Specifically, given a pattern $P$ of length $m$ and a text $T$ of length $n$, we want to find all occurrences of $P$ in $T$ that have no more than $k$ mismatches. The pattern can have don't care characters, which match any character. Without don't cares, the best known algorithm for pattern matching with $k$ mismatches has a runtime of $O(n \sqrt{k \log k})$. With don't cares in the pattern, the best deterministic algorithm has a runtime of $O(n k$ polylog $m)$. Therefore, there is an important gap between the versions with and without don't cares.

In this paper we give an algorithm whose runtime increases with the number of don't cares. We define an island to be a maximal length substring of $P$ that does not contain don't cares. Let $q$ be the number of islands in $P$. We present an algorithm that runs in $O\left(n \sqrt{k \log m}+n \min \left\{\sqrt[3]{q k \log ^{2} m}, \sqrt{q \log m}\right\}\right)$ time. If the number of islands $q$ is $O(k)$ this runtime becomes $O(n \sqrt{k \log m})$, which essentially matches the best known runtime for pattern matching with $k$ mismatches without don't cares. If the number of islands $q$ is $O\left(k^{2}\right)$, this algorithm is asymptotically faster than the previous best algorithm for pattern matching with $k$ mismatches with don't cares in the pattern.
\end{abstract}

Keywords: pattern matching with $\mathrm{k}$ mismatches and don't cares, $\mathrm{k}$ mismatches with wild cards, $\mathrm{k}$ mismatches with don't cares in the pattern

\footnotetext{
*Corresponding author

Email addresses: marius.nicolae@engr.uconn.edu (Marius Nicolae), rajasek@engr.uconn.edu (Sanguthevar Rajasekaran)
} 


\section{Introduction}

The problem of string matching can be defined as follows. Given a text $T=t_{1} t_{2} \cdots t_{n}$ and a pattern $P=p_{1} p_{2} \cdots p_{m}$, with letters from an alphabet $\Sigma$, find all the occurrences of the pattern in the text. This problem can be solved in $O(n+m)$ time by using well known algorithms (e.g., KMP [1]).

A more general formulation allows "don't care" or "wild card" characters in the text and/or the pattern. Pattern matching with don't cares can be solved in $O(n \log |\Sigma| \log m)$ as shown in [2]. A more recent result [3] gives a deterministic $O(n \log m)$ time algorithm.

Yet another enhancement is to allow for mismatches. We can formulate two versions of this problem: 1) pattern matching with mismatches: find the distance between the pattern and the text for every alignment between the pattern and the text or 2) pattern matching with $k$ mismatches: find only alignments for which the distance is no more than a given threshold $k$.

The distance metric used can be the Hamming distance, the edit distance or other criteria such as the number of non-overlapping inversions (e.g. [4]). In this paper we focus on the Hamming distance. The Hamming distance between two strings $A$ and $B$ is defined as the number of positions where the two strings differ and is denoted by $H d(A, B)$.

Pattern matching with mismatches can be solved, naively, by computing the Hamming distance for every alignment of the pattern in the text, in time $O(\mathrm{~nm})$. However, the fastest known exact algorithm is Abrahamson's algorithm [5] that runs in $O(n \sqrt{m \log m})$ time.

Pattern matching with $k$ mismatches can be solved in $O(n k)$ time (see [6] and [7]). These algorithms are based on a technique called the Kangaroo method (see section 2.1.4). This method computes the Hamming distance for every alignment in $O(k)$ time by "jumping" from one error to the next. A faster algorithm for pattern matching with $k$ mismatches runs in $O(n \sqrt{k \log k})$ [8]. A simpler version of this algorithm was given in [9].

Recent work has also addressed the online version of pattern matching, where the text is received in a streaming model, one character at a time, and it cannot be stored in its entirety (see e.g., [10], [11], [12]). Another version of this problem matches the pattern against multiple input streams (see e.g., [13]). Yet another interesting problem is to sample a representative set of mismatches for every alignment (see e.g., [14]). A survey of string matching with mismatches is given in [15]. A description of practical on-line string 
searching algorithms can be found in [16].

Yet another formulation allows for don't care or wild card characters. Pattern matching with mismatches and don't cares can be solved in $O(n \sqrt{g \log m})$ time, where $g$ is the number of non-wild card positions in the pattern (see [9]). This is done by a simple extension of Abrahamson's algorithm.

Pattern matching with $k$ mismatches and don't cares can be solved in time $O\left(n k^{2} \log ^{2} m\right)$ as shown in [17]. The runtime can be improved to $O(n k$ polylogm $)$ as shown in $[17,18]$ If we allow don't cares only in the pattern, the problem can be solved in $O\left(n \sqrt[3]{m k \log ^{2} m}\right)$ time as shown in [19]. This is also the problem we discuss in this paper.

Notation: Let $T_{i}$ denote $t_{i} t_{i+1}, \ldots t_{i+m-1}$ for all $i=1 . . n-m+1$.

Pattern matching with $k$ mismatches and don't cares in the pattern: Given a text $T=t_{1} t_{2} \ldots t_{n}$ and a pattern $P=p_{1} p_{2} \ldots p_{m}$ from an alphabet $\Sigma$, with $|\Sigma| \leq n$, and an integer $k$. Output all $i, 1 \leq i \leq n-m+1$, for which $H d\left(P, T_{i}\right) \leq k$. The pattern may contain don't care characters, that match any character.

Given a pattern $P$, with don't cares, a maximal length substring of $P$ that has no don't cares is called an "island". We will denote the number of islands in $P$ as $q$. In this paper we give two algorithms for pattern matching with $k$ mismatches where there are don't cares in the pattern. The first one runs in $O(n \sqrt{(q+k) \log m})$ time. The second one runs in time $O\left(n \sqrt[3]{q k \log ^{2} m}+\right.$ $n \sqrt{k \log m}$ ) where $q$ is the number of islands in $P$. By combining the two, we show that pattern matching with $k$ mismatches and don't cares in the pattern can be solved in $O\left(n \sqrt{k \log m}+n \min \left\{\sqrt[3]{q k \log ^{2} m}, \sqrt{q \log m}\right\}\right)$ time. If the number of islands is $O(k)$ our runtime becomes $O(n \sqrt{k \log m})$, which essentially matches the best known runtime for pattern matching with $k$ mismatches without don't cares $(O(n \sqrt{k \log k}))$. Since $q$ is always less than $m$, our algorithm outperforms the $O\left(n \sqrt[3]{m k \log ^{2} m}\right)$ algorithm of [19]. For $q=O\left(k^{2}\right)$, our algorithm outperforms the best known $O(n k$ polylog $m)$ algorithms of $[17,18]$.

\section{Methods}

Both algorithms in this paper have the same basic structure (see section 2.2). The difference is in how fast we can answer the single alignment verification question: 
Question 1. Given $i$, is the Hamming distance between $P$ and $T_{i}$ no more than $k$ ?

In the first algorithm (section 2.3), we can answer this question in $O(q+k)$ time. In the second algorithm (section 2.4), we can answer this question in $O\left(\sqrt[3]{k^{2} q^{2} \log m}+k\right)$ time.

\subsection{Background}

We start by reviewing a number of well known techniques used in the literature for pattern pattern matching with $k$ mismatches (e.g., see [8]), namely: convolution, marking, filtering and the Kangaroo method.

\subsubsection{Convolution}

Given two arrays $T=t_{1} t_{2} \ldots t_{n}$ and $P=p_{1} p_{2} \ldots p_{m}$ (with $m \leq n$ ), the convolution of $T$ and $P$ is a sequence $C=c_{1}, c_{2}, \ldots, c_{n-m+1}$ where $c_{i}=$ $\sum_{j=1}^{m} t_{i+j-1} p_{j}$, for $1 \leq i \leq(n-m+1)$.

Convolution can be applied to pattern matching with mismatches, as follows. Given a string $S$ and a character $\alpha$ define string $S^{\alpha}$ as $S^{\alpha}[i]=1$ if $S[i]=\alpha$ and 0 otherwise. Let $C^{\alpha}=\operatorname{convolution}\left(T^{\alpha}, P^{\alpha}\right)$. Then $C^{\alpha}[i]$ gives the number of matches between $P$ and $T_{i}$ where the matching character is $\alpha$. Therefore, one convolution gives us the number of matches contributed by

a single character to each of the alignments. Then $\sum_{\alpha \in \Sigma} C^{\alpha}[i]$ is the total number of matches between $P$ and $T_{i}$.

One convolution can be computed in $O(n \log m)$ time by using the Fast Fourier Transform. If the convolutions are applied on binary inputs, as is often the case in pattern matching applications, some speedup techniques are presented in [20].

\subsubsection{Marking}

Marking is an algorithm that counts the number of matches of every alignment, as follows. The algorithm scans the text one character at a time and "marks" all the alignments that would produce a match between the current character in the text and the corresponding character in the pattern. The marking algorithm is generally used only on a subset of the pattern. That is, given a set $A$ of positions in $P$ the marking algorithm counts matches between the text and the subset of $P$ given by $A$. The pseudocode of the marking algorithm is given in Algorithm 1. 


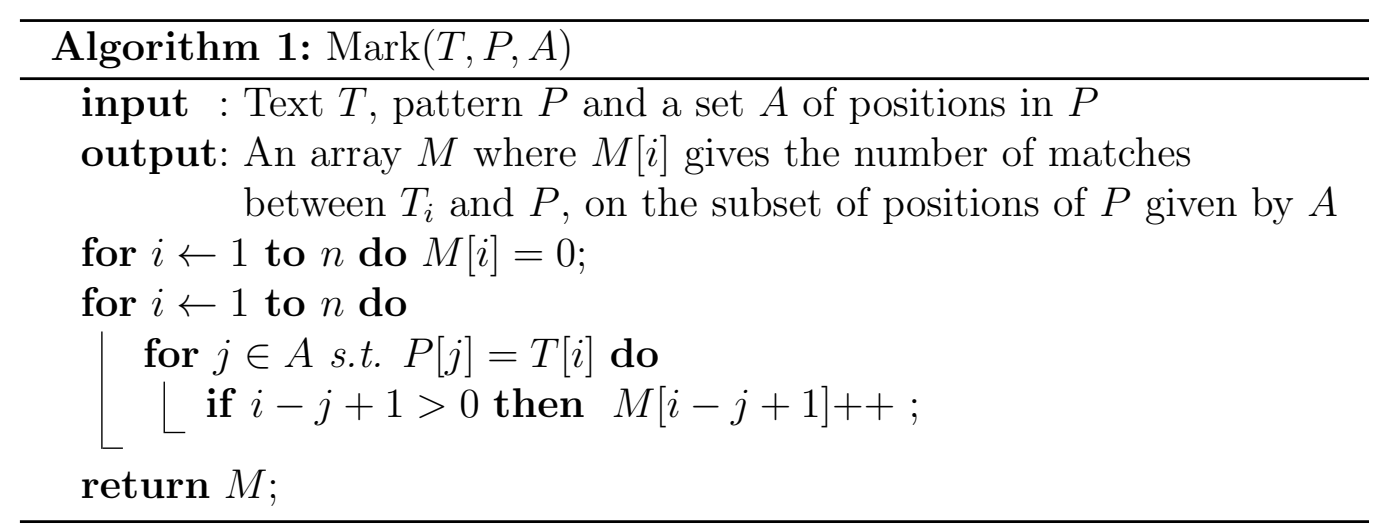

\subsubsection{Filtering}

Filtering is a method for reducing the number of alignments to look at. Filtering is based on the following principle. If we restrict our pattern to only $2 k$ positions, any alignment that has no more than $k$ mismatches, must have at least $k$ matches among the $2 k$ positions. To count matches among the $2 k$ positions selected, for every alignment, we use the marking algorithm. If the total number of marks generated is $B$ then there can be no more than $B / k$ positions that have at least $k$ marks. Therefore, instead of $n-m+1$ alignments we only have to look at $B / k$ alignments. Each alignment is then verified using other methods.

\subsubsection{The Kangaroo method}

The Kangaroo method allows us to check if the number of mismatches for a particular alignment is no more than $k$, in $O(k)$ time. The Kangaroo method constructs a generalized suffix tree of $T+P$, where + means concatenation. This suffix tree can be enhanced to answer Lowest Common Ancestor (LCA) queries in $O(1)$ time [21]. LCA queries give us the longest common prefix between any portion of the text and any portion of the pattern, essentially telling us where the first mismatch appears. Specifically, to count mismatches between $P$ and $T_{i}$, first perform an LCA query to find the position of the first mismatch between $P$ and $T_{i}$. Let this position be $j$. Then, perform another LCA to find the first mismatch between $P_{j+1 . . m}$ and $T_{i+j+1 . . i+m-1}$, which gives the second mismatch of alignment $i$. Continue to "jump" from one mismatch to the next, until the end of the pattern is reached or we have found more than $k$ mismatches. Therefore, after $O(k)$ LCA queries we will either find all the mismatches or determine that there 
are more than $k$ of them. The Kangaroo pseudocode is given in Algorithm 2 .

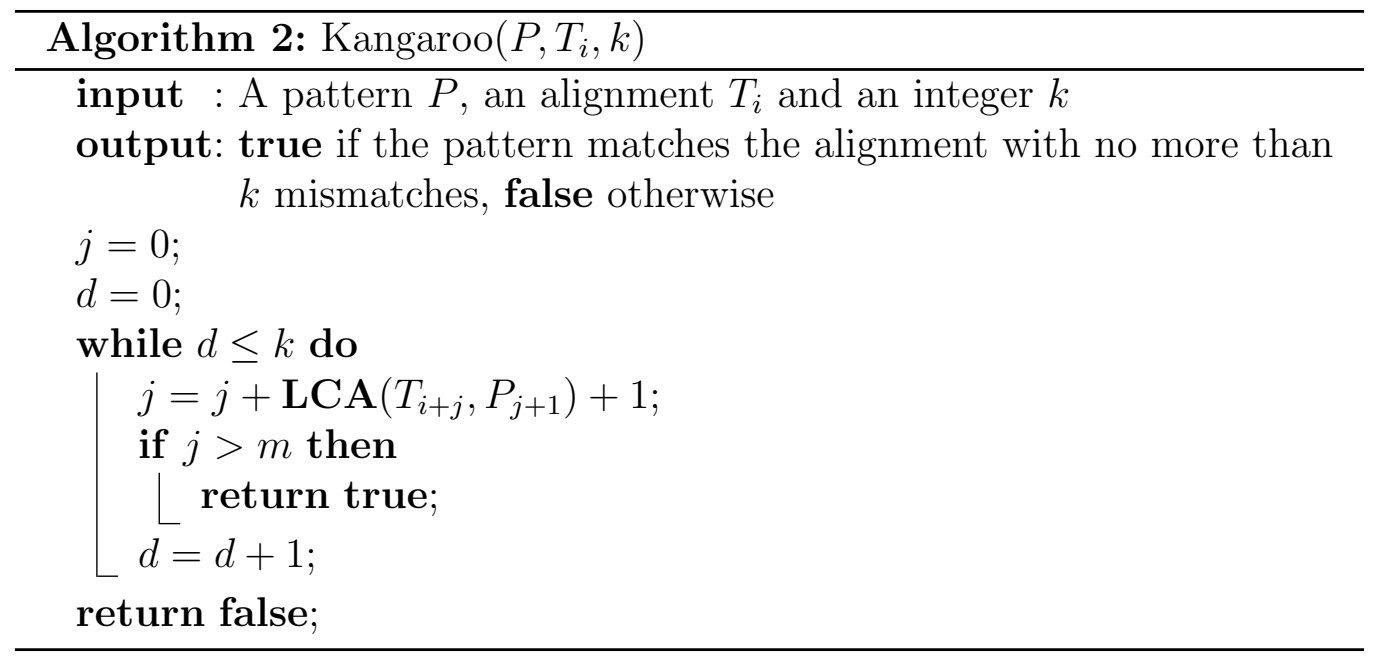

\subsection{General Algorithm}

We are now ready to present the main algorithms given in this paper. The general structure of both the algorithms is given in Algorithm 3.

Algorithm and analysis: For each position $i$ in $P$ such that $P[i]=a$, we assign a cost $F_{a}$ where $F_{a}$ is the number of occurrences of $a$ in $T$. The algorithm starts by choosing up to $2 k$ positions from the pattern such that the total cost does not exceed a "budget" $B$. The positions are chosen by a simple greedy strategy: sort all the characters by their cost $F_{a}$. Start choosing positions equal to the "cheapest" character, then choose positions equal to the next cheapest character, and so on until we have chosen $2 k$ positions or we have exceeded the budget $B$.

Case 1: If we can find $2 k$ positions that cost no more than $B$, then we call the marking algorithm with those $2 k$ positions. Any position in $T$ that receives less than $k$ marks, has more than $k$ mismatches, so we now focus on positions in $T$ that have at least $k$ marks. If the total number of marks is $B$, then there will be no more than $B / k$ positions that have at least $k$ marks. We verify each of these positions to see if they have more than $k$ mismatches. Let the time for a single verification be $O(V)$. Then, the runtime is $O(B V / k)$.

Case 2: If we cannot find $2 k$ positions that cost no more than $B$, then we compute marking for the positions that we did choose before we ran 


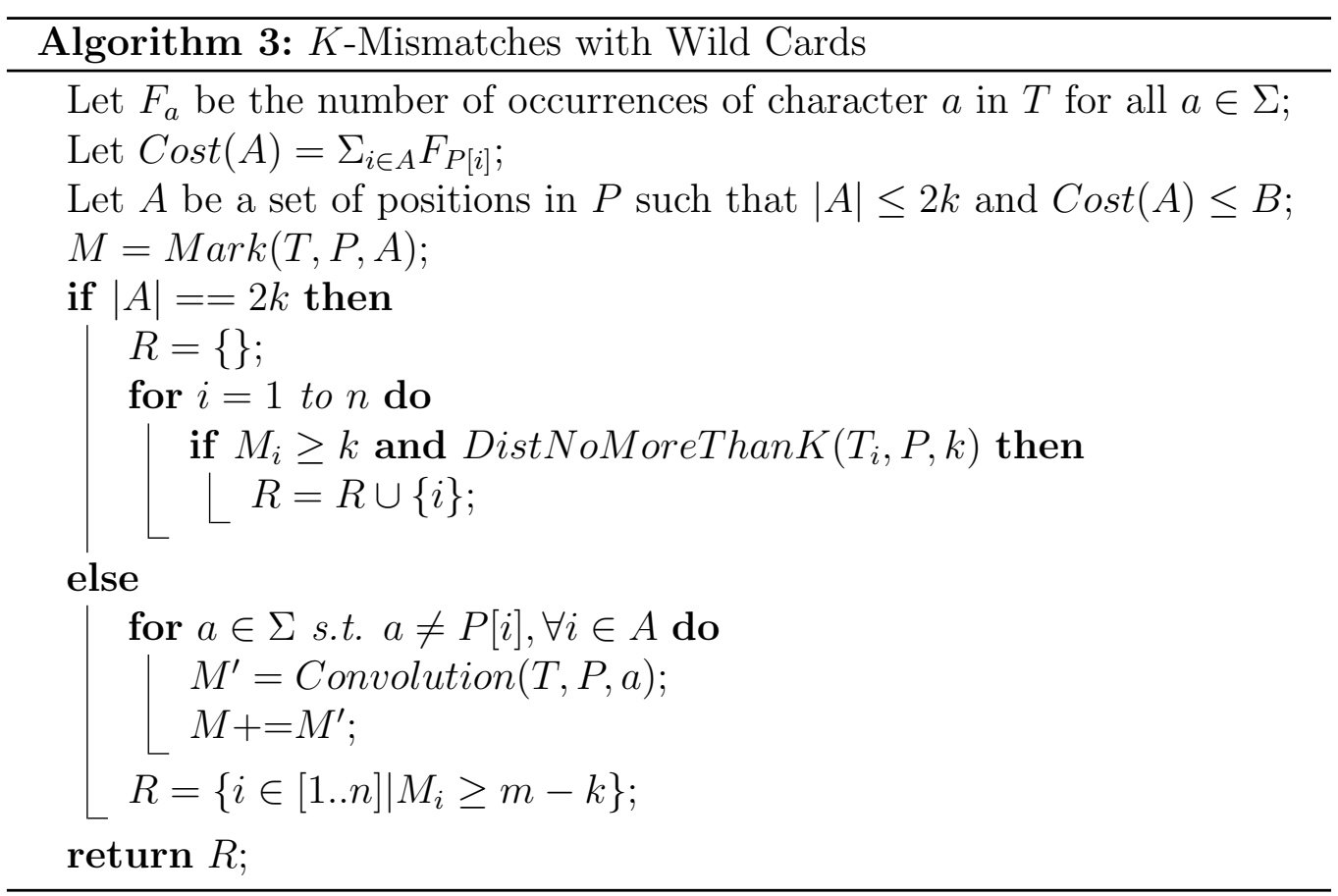

out of budget. Then, for each of the characters that we did not choose, we compute one convolution to count how many matches they contribute to each alignment. It is easy to see that each of the characters not chosen for marking must have $F_{a}>B /(2 k)$. Therefore, the total number of such characters is no more than $n /(B /(2 k))$. Therefore, the runtime of the convolution stage is $O(n k / B * n \log m)$. The runtime of the marking stage is $O(B)$, therefore the total runtime is $O(B+n k / B * n \log m)$.

If we make the runtime of the two cases equal, we can find the optimal value of $B$.

$$
B V / k=B+n^{2} k / B \log m \Rightarrow B=n k \sqrt{\frac{\log m}{V}}
$$

This gives an asymptotic runtime of $O(B V / k)=O(n \sqrt{V \log m})$. Therefore, the runtime of the algorithm depends on $V$, which is the time it takes to verify whether a given single alignment has no more than $k$ mismatches. 


\subsection{Single alignment distance in $O(q+k)$ time}

We can answer the single alignment question in $O(q+k)$ time where $q$ is the number of islands in the pattern as shown in Algorithm 4. The algorithm uses Kangaroo jumps [6] to go to the next mismatch within an island in $O(1)$ time. If there is no mismatch left in the island, the algorithm goes to the next island also in $O(1)$ time. Therefore, the runtime is $O(q+k)$. With $V=O(q+k)$, Algorithm 3 does pattern matching with $k$ mismatches in $O(n \sqrt{(q+k) \log m})$ time.

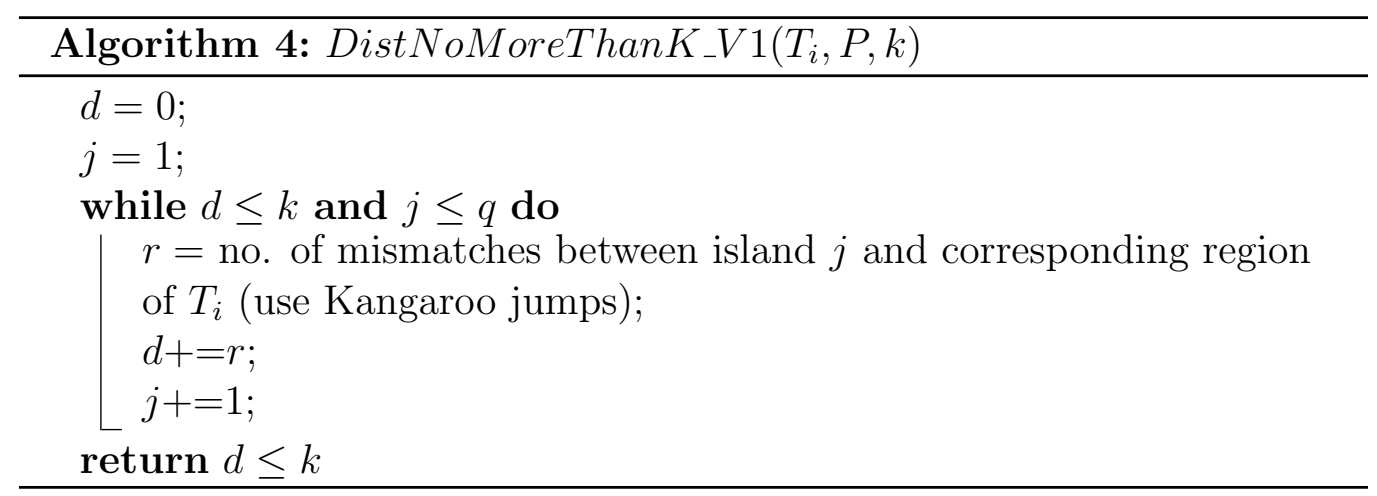

\subsection{Single alignment distance in $O\left(k^{2 / 3} q^{2 / 3} \log ^{1 / 3} m+k\right)$ time}

This idea is based on splitting the pattern into sections. We know that no more than $k$ sections can have mismatches. The remaining sections have to match exactly. Consider exact pattern matching with don't cares. We can check where a pattern matches the text exactly by using a constant number of convolutions. This is true because we can compute the values $C_{i}=\sum_{j=0}^{m-1}\left(T_{i+j}-P_{j}\right)^{2} T_{i+j} P_{j}$ using a constant number of convolutions (see [3]). If $C_{i}=0$ then the pattern matches the text at position $i$.

Using this result, we will split the pattern into $S$ sections. In each section we include $q / S$ islands. For each of the $S$ sections, we use a constant number of convolutions to check where the section matches the text. If $P$ has no more than $k$ mismatches at a particular alignment, then at least $S-k$ sections have to match exactly. Each of the at most $k$ sections that do not match exactly are verified using Kangaroo jumps as seen earlier. One section takes at most $O\left(q / S+k^{\prime}\right)$ time, where $k^{\prime}$ is the number of mismatches discovered in that section. Over all the sections, the $k^{\prime}$ terms add up to no more than $k$, therefore the entire alignment can be verified in time $O(S+k+k q / S)$. 
If we make $V=O(S+k+k q / S)$ in Algorithm 3, then its runtime becomes $O(n \sqrt{V \log m})=O(n \sqrt{(S+k+k q / S) \log m})$. The preprocessing time for the $S$ sections is $O(S n \log m)$. The optimal value of $S$ is such that the preprocessing equals the main runtime:

$$
\begin{aligned}
& n \sqrt{(S+k+k q / S) \log m}=S n \log m \\
\Rightarrow & S+k+k q / S=S^{2} \log m \\
\Rightarrow & S^{2} / \log m+k S / \log m+k q / \log m=S^{3} \\
\Rightarrow & S \approx O(\sqrt[3]{k q / \log m})
\end{aligned}
$$

This makes $V=O(S+k+k q / S)=O\left(k+\sqrt[3]{k^{2} q^{2} \log m}\right)$. This gives a runtime for pattern matching with $k$ mismatches of:

$$
\begin{aligned}
O(n S \log m+n \sqrt{V \log m}) & =O\left(n \sqrt[3]{k q \log ^{2} m}+n \sqrt{\left(k+\sqrt[3]{k^{2} q^{2} \log m}\right) \log m}\right) \\
& =O\left(n \sqrt[3]{k q \log ^{2} m}+n \sqrt{k \log m}\right)
\end{aligned}
$$

\subsection{Combined result}

If $q<k^{2}$ then we can use the algorithm of section 2.3, which runs in $O(n \sqrt{(q+k) \log m})$ time. Otherwise, if $q>k^{2}$, we use the algorithm of section 2.4, which runs in $O\left(n \sqrt[3]{q k \log ^{2} m}+n \sqrt{k \log m}\right)$ time. Thus we have the following:

Theorem 1. Pattern matching with $k$ mismatches, with don't care symbols in the pattern, can be solved in $O\left(n \sqrt{k \log m}+n \min \left\{\sqrt{q \log m}, \sqrt[3]{q k \log ^{2} m}\right\}\right)$ time.

\section{Conclusions}

In this paper we have offered efficient algorithms for the problem of pattern matching with $k$ mismatches. Specifically, we have presented an algorithm that runs in $O\left(n \sqrt{k \log m}+n \min \left\{\sqrt[3]{q k \log ^{2} m}, \sqrt{q \log m}\right\}\right)$ time, where $q$ is the number of islands. If the number of islands $q$ is $o(m)$, this algorithm is asymptotically faster than the previous best algorithm for pattern matching with $k$ mismatches with don't cares in the pattern. 


\section{Acknowledgments}

This work has been supported in part by the following grants: NSF 1447711 and NIH R01-LM010101.

\section{Bibliography}

[1] D. E. Knuth, J. James H. Morris, V. R. Pratt, Fast pattern matching in strings, SIAM Journal on Computing 6 (2) (1977) 323-350. doi: $10.1137 / 0206024$.

[2] M. J. Fischer, M. S. Paterson, String-matching and other products, Tech. Rep. MAC-TM-41, Massachusetts Institute of Technology Cambridge Project MAC, Cambridge, MA, USA (1974).

[3] P. Clifford, R. Clifford, Simple deterministic wildcard matching, Information Processing Letters 101 (2) (2007) 53 - 54. doi:10.1016/j.ipl.2006.08.002.

[4] D. Cantone, S. Cristofaro, S. Faro, Efficient string-matching allowing for non-overlapping inversions, Theoretical Computer Science 483 (2013) 85-95. doi:10.1016/j.tcs.2012.06.009.

[5] K. Abrahamson, Generalized string matching, SIAM Journal on Computing 16 (6) (1987) 1039-1051. doi:10.1137/0216067.

[6] G. M. Landau, U. Vishkin, Efficient string matching in the presence of errors, in: Foundations of Computer Science, 1985., 26th Annual Symposium on, IEEE, 1985, pp. 126 -136. doi:10.1109/SFCS.1985.22.

[7] Z. Galil, R. Giancarlo, Improved string matching with k mismatches, SIGACT News 17 (4) (1986) 52-54. doi:10.1145/8307.8309.

[8] A. Amir, M. Lewenstein, E. Porat, Faster algorithms for string matching with k mismatches, J. Algorithms 50 (2) (2004) 257-275. doi:10.1016/S0196-6774(03)00097-X.

[9] M. Nicolae, S. Rajasekaran, On string matching with mismatches, Algorithms 8 (2) (2015) 248-270. doi:10.3390/a8020248. 
[10] R. Clifford, K. Efremenko, B. Porat, E. Porat, A black box for online approximate pattern matching, in: Combinatorial Pattern Matching, Springer-Verlag, 2008, pp. 143-151.

[11] B. Porat, E. Porat, Exact and approximate pattern matching in the streaming model, in: Foundations of Computer Science, 2009. FOCS '09. 50th Annual IEEE Symposium on, 2009, pp. 315-323. doi:10.1109/FOCS.2009.11.

[12] E. Porat, O. Lipsky, Improved sketching of hamming distance with error correcting, in: Combinatorial Pattern Matching, Springer, 2007, pp. $173-182$.

[13] R. Clifford, K. Efremenko, E. Porat, A. Rothschild, k-mismatch with don't cares, Algorithms-ESA 2007 (2007) 151-162.

[14] R. Clifford, K. Efremenko, B. Porat, E. Porat, A. Rothschild, Mismatch sampling, Inf. Comput. 214 (2012) 112-118. doi:10.1016/j.ic.2012.02.007.

[15] G. Navarro, A guided tour to approximate string matching, ACM Comput. Surv. 33 (1) (2001) 31-88. doi:10.1145/375360.375365.

[16] G. Navarro, M. Raffinot, Flexible pattern matching in strings - practical on-line search algorithms for texts and biological sequences, Cambridge University Press, 2002.

URL http://www.dcc.uchile.cl/ gnavarro/FPMbook/

[17] R. Clifford, K. Efremenko, E. Porat, A. Rothschild, Pattern matching with don't cares and few errors, Journal of Computer and System Sciences 76 (2) (2010) 115 - 124. doi:10.1016/j.jcss.2009.06.002.

URL http://www. sciencedirect. com/science/article/pii/S0022000009000567

[18] R. Clifford, K. Efremenko, E. Porat, A. Rothschild, From coding theory to efficient pattern matching, in: Proceedings of the Twentieth Annual ACM-SIAM Symposium on Discrete Algorithms, SODA '09, Society for Industrial and Applied Mathematics, Philadelphia, PA, USA, 2009, pp. 778-784.

URL http://dl.acm.org/citation. cfm?id=1496770.1496855 
[19] R. Clifford, E. Porat, A filtering algorithm for k-mismatch with dont cares, in: String Processing and Information Retrieval, Springer, 2007, pp. 130-136.

[20] K. Fredriksson, S. Grabowski, Combinatorial algorithms, SpringerVerlag, Berlin, Heidelberg, 2009, Ch. Fast Convolutions and Their Applications in Approximate String Matching, pp. 254-265.

[21] A. V. Aho, J. E. Hopcroft, J. D. Ullman, On finding lowest common ancestors in trees, SIAM Journal on computing 5 (1) (1976) 115-132. doi:10.1137/0205011. 